Haggis, T. (2008) ' Knowledge must be contextual': exploring some possible implications of complexity and dynamic systems theories for educational research Educational Philosophy and Theory Vol 40 No 1. pp 159-176

\title{
'Knowledge must be contextual': Some possible implications of complexity and dynamic systems theories for educational research
}

\author{
Tamsin Haggis, University of Stirling, Scotland
}

\begin{abstract}
It is now widely accepted that qualitative and quantitative research traditions, rather than being seen as opposed or in competition with each other (Hammersley \& Atkinson, 1995; Furlong, 2004) should be used, where appropriate, in some kind of combination (Bryman \& Cramer, 1999; Moore et. al. 2003). How this combining is to be understood ontologically, and therefore epistemologically, however, is not always clear. After looking briefly at the wide variety of different practices and epistemologies which are conflated in the use of qualitative/quantitative as a binary concept, the paper will explore this area of 'critical connections' in relation to the analysis of data. Complexity/dynamic systems theory will be explored as offering the basis of a different kind of ontology which might open up possibilities for creating different types of knowledge (see Byrne, 1997).
\end{abstract}

\section{HOW IS THE COMBINING OF QUALITATIVE/QUANTITATIVE METHODS TO BE UNDERSTOOD?}

The assumption that qualitative and quantitative traditions can be 'fused' (Bryman \& Cramer, 1999), or at least combined meaningfully (for example, in mixed method approaches), raises a number of questions about how such fusion/combining is understood, in terms of the meaning and interpretation of different kinds of research results (whether these are based on realist, interpretive or other types of philosophical orientation). Although some researchers seem to suggest that the relationship between these different approaches to research is relatively unproblematic (eg. Silverman, $2001^{1}$;), others claim that the different approaches start from contradictory ontological, epistemological (Yanow, 2003) and philosophical (Clark, 2005) bases, resulting in a tendency for theorising to 'remain bifurcated' (Flybverg, 2001:138), and for social scientists to 'tend to generate macrolevel or microlevel explanations' whilst 'ignoring the critical connections' (ibid).

As a binary category, quantitative/qualitative is intrinsically problematic, referring to a wide range of different methods which can be allied to many different intentions and philosophical orientations. 'Qualitative' can refer, amongst other things, to 'small n' studies in psychology (where a case study is in effect a smaller unit of focus for a largely numerical approach) as well as to various types of 'ethnographic study' in sociology or anthropology (where the case study attempts to elucidate a complexity of meanings and interpretations). In terms of method, interview data can be treated quantitatively (Strauss \& Corbin, 1998), in the sense that occurrences of words or concepts may be counted, and statistical data collection may include qualitative elements (such as open-ended questions in a questionnaire). Qualitative data which attempts to elucidate meanings can also be analysed in a variety of ways: using semiotic analysis in relation to 'thick descriptions' (Geertz, 1973); analytic induction in relation to increasing numbers of single cases (Smelser \& Bates, 2001; Hammersley, 1989); or 'constant comparative analysis' (Strauss \& Corbin, 1998), to name but a few different approaches. In addition, qualitative analysis may be

\footnotetext{
${ }^{1}$ Although Silverman elsewhere warns against a simplistic view of multiple methods, here he states that 'the proper relationship' between quantitative and qualitative work 'is a division of labour in which qualitative researchers seek to answer 'how' and 'what' questions and then pass on their findings, so that causes and outputs of the phenomena identified ('why' questions) can be studied by their quantitative colleagues' (2001:259)
} 
underpinned by realist, interpretive or postmodern assumptions and/or intentions, which all produce different kinds of results in relation to bodies of data (Yanow, 2003).

\section{'CRITICAL CONNECTIONS'?}

The different, and possibly contradictory, philosophies/ontologies which can underpin different approaches to research has been discussed by many (eg. Hammersley, 1989). Rock's distinction between 'phenomenalism ('the need to faithfully reproduce the social world as it is known by its inhabitants', 1973:17) and 'essentialism' ('the search for the underlying properties of social order', ibid.) suggests one way of understanding what might also be framed as a distinction between a desire for explanation and a desire for understanding (Hollis, 2003). Whilst some researchers appear to simply accept the existence of different research intentions as different, (Law \& Urry, 2003), others are stimulated to attempt to bridge or reconcile such differences, as, for example, in grounded theory's attempt to create a 'genuine interplay' between qualitative and quantitative approaches (Strauss \& Corbin, 1998).

In these 'recurrent discussions about the relationship between qualitative and quantitative work' (Hammersley, 2005), is the choice, though, only between attempting to reconcile different philosophical orientations and/or purposes, or accepting that they may be unreconcilable? Perhaps, alternatively, there is something to 'unearth' here, in relation to more fundamental and largely invisible cultural orientations. It has been argued, for example, that approaches which declare themselves to be distinct theoretically are often surprisingly similar methodologically, in the sense that many declaredly interpretive types of qualitative research are informed by the principles which underpin realist, statistical approaches. Thomas (2002), drawing on Stronach, suggests that 'the search for theory in qualitative research is characterised by an unqualitative approach to qualitative enquiry' (2002:430), and claims that naturalist ethnography takes a 'neopositivist stance' which represents a 'modernist pursuit of a post-modern goal'(ibid: 422). In the same vein, Law and Urry (2003) suggest that:

method in practice, whatever its theoretical stance, and whatever its particular research tools, tends to a kind of empiricist realism: the assumption that in any given context and given the purposes of the study there is a single reality... (2003:13)

\section{Prevailing epistemologies: similarity categories, key factors, and deep structure}

The first way in which this empiricist realism is demonstrated is in the assumption that in order to make sense of data it is necessary to create abstractions which transcend the complex particularity of the data in specific ways. Although abstraction could, theoretically, be created in relation to the longitudinal history of each sub-unit within the case, the data is instead usually analysed cross-sectionally, on the assumption that comparison between different sub-cases will lead to the identification of key elements or themes which will have some meaning beyond each individual sub-history:

(Analytic) concepts may be 'observer identified'.... the analyst...(draws together) under the aegis of a single type what for members is a diverse and unrelated range of phenomena

Hammersley \& Atkinson, 1995; 211

In the analysis of narratives, for example, the smaller unit within a case is often the individual, with the comparative analysis taking place in relation to what can be seen to be shared across different narratives when these are viewed in relation to each other. In approaches such as grounded theory (Strauss \& Corbin, 1998) or phenomenography ${ }^{2}$ (Marton et al, 1997), the smaller unit is not necessarily the individual, but the overall intention is the same; to look across different elements of data and find a pattern of similarity which appears to transcend (and there for, at

\footnotetext{
${ }^{2}$ This refers to the influential body of work in Higher Education which describes 'deep' and 'surface' approaches to learning
} 
some kind of deeper level, to unite) these smaller units. The creation of such categories of 'things held in common' (eg. correlations, 'key factors', typologies, hierarchies) occurring in both quantitative and qualitative types of analysis, is fundamental to research in the social sciences (Llewlyn, 2003).

In creating a similarity pattern the researcher is, in effect, trying to 'see through' difference and variety to 'what lies beneath', in order to try to get a sense of generative principles (Gomm \& Hammersley, 2001). This is arguably a search for a subtle form of 'deep structure'. Whether or not such structure is seen instrumentally ${ }^{3}$, or in more realist terms, the implication in both cases is that its identification will lead to the possibility of other manifestations of variety becoming, at least in theory, more predictable. This approach certainly does identify meaningful patterns; patterns which do often relate to other, similar situations; and which can be successful in terms of facilitating certain kinds of generalisation and prediction (developments in science and technology show how productive an approach this can be). What is of interest here, however, is the observable reality that possibilities of generalisation and prediction based upon this approach are often limited when attempts are made to use it in relation to human and social phenomena (Gomm \& Hammersley, 2001; Byrne, 2005a). One way in which attention has been drawn to these limitations is the recent criticisms of educational research (Tooley \& Darby; 1998; Hargreaves, 1996). Whether or not such limitations are the 'problems' or 'failings' that this critique suggests is a matter of debate, but the existence of the debate itself points to some problematic areas in relation to epistemology.

Limitations in applying this approach to social phenomena are also suggested by the theoretical shift in fields such as anthropology and sociology towards an interest in difference, particularity, and local, contextual concerns. This shift accommodates a sensitivity to areas which conventional approaches are forced to downplay, such as time, process and connectivity. It has been suggested that approaches based on the prevailing ontology are not able to deal well with 'the fleeting', 'the distributed', 'the multiple' and 'the complex' (Law \& Urry, 2003:10). Such concerns have impacted on the development of educational theory to some extent, but these effects do not usually extend to an examination or questioning of the ontological and epistemological assumptions which underpin the practices of educational research, particularly in relation to methods of analysis. Though there have been critiques of these assumptions from postmodern and feminist perspectives, these tend to be based on approaches from areas such as literary theory and the visual arts. Complexity theory, on the other hand, offers a way of thinking about these issues from a perspective closer to the theoretical orientation of social scientists, and appears to open up a different way of thinking about individuals, classes, groups, cultures, and societies.

\section{PROBLEMS CONCEPTUALISING AND RESEARCHING DIFFERENCE, SPECIFICITY AND CONTEXT}

The theoretical shift in the social sciences towards an interest in understanding 'things in context' implies the need to investigate difference and particularity. This is arguably of particular relevance to educational research, as it attempts to create knowledge which can be used in relation to practices in specific contexts But practices, and people, in specific contexts are particularly difficult to investigate from the dominant epistemological, and ontological, position.

Many forms of case study research do, of course, make 'people in context' the focus of their investigations. Different types of qualitative study focus on small groups of individuals, attempting to capture the richness of individual experience, and to understand how meaning functions and is made. But these studies arguably face something of a problem when it comes to writing up their results. The dominant ontology demands that it has to be possible to relate some aspect of the particular study to other, similar situations, but researchers at the same time know that they are not able to generalise from small samples. The final sections of research reports are often thus forced to present a kind of conceptual 'fudge', which may involve discussion of such things as 'lessons to be drawn', the possibility of 'illuminating' problems (Newton, 2003), or the drawing of

\footnotetext{
3 ،...the issue of realism and instrumentalism...is typically understood as the question of whether various terms in statements making up scientific theories refer to real objects or merely serve the role of facilitating inferences among claims about observations' (Giere, 2000: 515)
} 
conclusions that 'might apply' to other situations (Stein et al, 2004). Although qualitative case study researchers are usually careful to make it clear that they cannot generalise, this caveat itself indicates how such research is still firmly located within particular ontological and epistemological assumptions which privilege the capacity for a particular kind of generalisation. Clearly, some kind of connection between the results of a case study and other potential situations is possible, but these connections are subtle, interpretative, and to some degree speculative. They are not always convincing to funders of educational research, and do not really overcome the problem of how such subtle forms of connection may be understood to relate to the kinds of patterns described by other, more quantitative (eg. survey-based) forms of investigation.

A different approach to these problems is suggested by forms of analysis such as thick description' (Geertz, 1973) and 'analytic induction' (Smelser \& Bates, 2001; Roberts, 2002), both of which take the individual case as their starting point, rather than trying to stand back from a range of different cases and decide what they have in common. These approaches, however, although different from each other, both still reflect aspects of the approach discussed above, in that the aim is to gradually identify a principle which will be able to explain, and hopefully to predict aspects of, future, different occurrences of similar cases. Though these approaches are 'bottom up' (starting with concrete, individual cases), rather than 'top down' (looking across a range of individuals or elements within the case), the idea that a given phenomenon can be characterised in this way is based on the assumption that the only way to understand a phenomenon is to try to define central/underpinning tendencies or mechanisms (which ultimately pertain to the 'class' that the case is assumed to be an instance of).

Cross-sectional and comparative analysis clearly does identify important types of pattern and linkage. These forms of analysis can describe what is amenable to description in terms of variables or categories, and what is amenable to some form of counting or measurement. There are other aspects of the data, though, which cannot be described in relation to either of these areas. Firstly, what is different between the various transcripts obviously becomes invisible, in the sense that only what is similar can become a category or theme. Secondly, in order to create a theme, the focus of attention has to be named, boundaried, and removed from the complex web of its 'own' contexts. Thirdly, and linked to the previous points, the focus on articulating key aspects of phenomena means that many aspects of the data have to be disregarded. Fourthly, it is difficult to conceptualise time and process using this approach. Fifthly, this overall approach is underpinned by a desire to infer causal processes, despite the fact that these can never be measured directly.

In relation to the first two, difference and local context, Guba \& Lincoln (1998) discuss how what they call 'context-stripping' removes other variables in the context of the research which could 'greatly alter findings' if they were 'allowed to exert their effects' (197), a point also made by Strathern (1992) in relation to the analysis of anthropological data. In more qualitative types of data analysis, 'context-stripping' not only removes the details of individual lives and histories, but also aspects of the data which could disturb the analyst's creation of apparent regularities (Walsh, 1994:24 in Ashworth \& Lucas, 2000:298). The aggregated, unifying category or theme is created at the expense of the acknowledgement of other, less easily-disciplined situational factors which it could be argued are nonetheless crucial, either in making what is being examined functional, in realist/explanatory terms, or meaningful, in more interpretative terms. This is arguably partly the cause of some of the problems which can be experienced when attempts are made to 'apply' a general principle created in this way to a particular case, in the sense that the specifics of the situation can appear to refute, confound, or at least complicate such application.

Part of the problem here seems to be the way that context is conceptualised in relation to the boundaries of the case, and the relationship of this conceptualisation to the contexts of the individual sub-units within the case. If the intention is to 'interpret meanings in context', then comparative analysis of different interview narratives from a particular context (eg. a group of access students, an adult education class, etc.) appears to make this possible. The meanings which are represented by the interview transcripts, however, in one sense do not so much relate to the group or class which has been defined as the case, but rather to the local contexts inhabited by the different individuals who have been interviewed. In terms of generative forces, it 
is arguably these individual contexts (which include but also go beyond the membership of the defined group or class) which have created the meanings expressed in the narratives.

A similarity pattern created through comparative analysis of different interview texts (eg. a 'theme', or group of themes) arguably says more about the group which has been defined as the case than it does about the individuals within the group. Paradoxically, however (given that individual contexts are not considered in the analysis), the theme is far more likely to be presented as information about the individuals ('these adults are all motivated by career prospects' rather than 'this university setting, in the context of current political and cultural agendas, encourages these adults to talk about learning in terms of career prospects). This kind of theme is often presented as if it points towards some kind of 'deep structure' which might help to explain the diversity of individuals. An example of this is Goodwin's (2002) study of adult learners at university, which identifies three categories of individual: 'pleasers', 'searchers' and 'skeptics'. These transcendent categories appear to unify certain aspects of the different narratives which have been analysed, although all of these were generated from within very different contextual settings.

Whether or not this is problematic depends on the purpose of the research. If the researcher is trying to understand how individuals within the context defined as the case understand that particular context (eg. the university class), then comparative analysis of smaller units, from one perspective at least, makes this possible ${ }^{4}$. Similarly, if the researcher wanted to investigate how larger discourse patterns transcend the individual contexts within which language is generated, this could also be done using comparative analysis. But if the researcher was trying to understand individual experience, to 'give voice' to individual perspectives, then the comparative analysis of interview texts seems to contradict this intention. Describing a particular group as a case allows for patterns which transcend individual uniqueness to be identified, but it does not allow any examination of the ways in which individuals are, in some aspects, also unique. Each individual within a case could, from a different perspective, be seen as a case in themselves, but current epistemologies do not really provide any way of conceptualising either the unique individual (other than as an example of a larger class) or of investigating uniquely individual differences in a meaningful way.

The third issue, that of having to disregard certain aspects of the data because of the assumption that it is necessary to articulate 'key aspects' of phenomena, arguably reflects what Thomas (2002), citing Derrida, refers to as a 'desire for center in the constitution of structure' (430). By definition, what is 'key' has to be separated out from a complexity of other factors which are deemed to be less important. But what if all of the factors in a particular situation were equally important, or if something that was determined to be unimportant was actually creating an effect on the phenomena being studied (a realist question, but one which works equally well if asked from a more interpretative stance...)? This possibility could not be accommodated within dominant conceptual frameworks. The reason that much of social life 'escapes our capacity to make models of it' (Law \& Urry, 2003: 7) could be because it is currently difficult to imagine and discuss a world in which multiple factors might be interacting 'equally', rather than in relation to definable 'driving forces' (not that imagining such multi-factor interactions would necessarily make it easier to model the world, but it might make it easier to talk about aspects of social life).

In relation to the fourth issue, difficulties conceptualising time and process, the removal of 'spatial and temporal grounding' (Nespor, 1994: 152) is an important casualty of the necessary eradication of 'contextual imperatives' (Unwin et al, 2005). Although interview studies may sometimes gather data at two or three different points in time, the range of conceptual resources available for the discussion of fluidity and change in relation to process and interaction is currently limited (Seibt, 2003), and this limitation is enhanced by cross-sectional methods of analysis (Stehr \& Grundmann, 2001).

\footnotetext{
${ }^{4}$ Students in an class, for example, could all comment on what they thought was happening: how the interaction was working, what the teacher was teaching, what aspects of the subject were difficult, etc.
} 


\section{Complexity theory: redefining order (and chaos)?}

Complexity theory (Cilliers, 1998; Byrne, 1998), sometimes also referred to as dynamic systems theory (Fogel. et. al, 1997; Valsiner, 1998), or as theories of emergence (Goldstein, 2000; Johnson, 2001), appears to provide a different way of conceptualising the issues so far discussed. 'Complexity theory' does not refer to a specific body of literature. Originating in the mathematical sciences, its ideas have been taken up in fields as diverse as archaelogy, law, philosophy and management. Richardson and Cilliers (2001), in a overview of the many uses of complexity theory, define what they call three themes, or communities, in the literature: 'hard, reductionist complexity science' (which aims to understand the principles of complex systems), 'soft complexity science' (which uses complexity as a metaphorical tool to understand organisations) and 'complexity thinking' (which considers the epistemological implications of assuming 'the ubiquity of complexity' (2001). More recently Byrne (2005a) has distinguished between 'simplistic' complexity (similar to the first of Richardson and Cillier's categories) and 'complex' complexity (which seems related to Richardson and Cillers third category, discussed in the context of research methodology). The use of complexity theory here is related to this last category, with a focus particularly on ontology. As a set of ideas about process and formation which can be used in relation to both realist/explanatory and interpretive approaches, complexity appears to offer not just another theory, but a completely different starting point for theory, and also for the conceptualisation of method.

Not everything that is complicated is seen to manifest features of 'complexity'. Cilliers (1998) distinguishes between 'complicated' (having many parts, but each part can be explained - eg a mechanical engine) and 'complex' (having many parts, but not all of which can be named, and not all processes involved can be tracked or described). With regard to the complex, Johnson (2001), following Weaver (1948 in Johnson, 2001), suggests that there are three types of scientific enquiry. The first deals with problems involving very limited numbers of variables, and concerns issues such as the movement of the planets around the sun (the approach underpinning Newtonian mechanics). The second approach deals with problems with are characterised by 'millions or billions of variables that can only be approached by the use of statistical mechanics and probability theory' (2001:46), which he calls 'disorganised complexity'. He suggests, however, that there is a field between these two approaches which deals with a still substantial number of variables, but with one crucial difference:

...much more important than the mere number of variables is the fact that these variables are all interrelated... these problems, as contrasted with the disorganised situations with which statisticians can cope, show the essential feature of organisation. We will therefore refer to this group of problems as those of organised complexity.

\section{Weaver, 1948, in Johnson, 2001: 47 (italics in original)}

Much large-scale social science research could be conceptualised as attempting to deal with 'disorganised complexity'. However, although the complexity of the social world, taken as a whole, could be conceptualised as being characterised by 'millions or billions of variables', such complexity could also be conceptualised as consisting of a large number of smaller, overlapping types of 'organised' (but open) systems. Cultures, discourses, practices, social groupings, institutions, and individuals could all be seen as 'open systems' which manifest different types of organisation.

In terms of the methods used to investigate such systems, cross-sectional and comparative types of analysis (whether in large-scale surveys or qualitative case studies) function by deliberately discounting the relationships which exist within the smaller units bounded by the population or case (eg. people, classes, schools), in order to focus on the patterns which can be seen when these smaller units are viewed in relation to each other. The interconnectedness of the elements within these types of units is seen to be too specific to be useful for the purpose of extracting a general principle. By contrast, dynamic systems theories focus upon the interactions and relationships that occur within open systems, however the idea of 'system' may be applied. 
A dynamic system is seen to consist of a large number of components which are interacting dynamically, at a local level (Cilliers, 1998). These multiple interactions are non-linear, involving complex feedback loops which continually adjust and modify both the 'parts' of the system, and the system itself. As the system is open, the interactions can also affect the boundaries of the system itself, and indeed have effects beyond it. Moreover, because the interactions are always local, such effects are distributed, rather than emanating from any central cause (Osberg, 2002; Johnson, 2001). If there is a sufficient number of these interactions, and if they take place over a sufficiently long period of time, specific forms of order, or organisation, will periodically emerge from within the system (Johnson, 2001). An example of this discussed by Johnson is the way that neighbourhoods within cities organise themselves around social class, and the way that cities themselves continually change and adapt in ways that have not been planned:

Cities have no central planning commissions that solve the problem of purchasing and distributing supplies... How do these cities avoid devastating swings between shortage and glut, year after year, decade after decade? The mystery deepens when we observe the kaleidoscopic nature of large cities. Buyers, sellers, administrators, streets, bridges and buildings are always changing, so that a city's coherence is somehow imposed on a perpetual flux of people and structures. Like the standing wave in front of a rock in a fast-moving stream, a city is a pattern in time.

Holland, 1998, in Johnson, 2001:27

Discussion of the unpredictability of emergence in this kind of description of dynamic processes is often misunderstood to imply randomness, chaos or non-determinism. Non-linear systems, however, are in fact understood, at least in some ways, to be deterministic, although the idea of determinism is perhaps differently nuanced in this context. There is causality, but not of the 'a causes b' kind. For some writers properties 'emerge deterministically from non-linear rules of interaction', and are thus 'merely unexpected' in relation to 'the principles governing the lowerlevel domain' (Osberg, 2005:169). For others, however, emergence suggests a more radical kind of novelty which cannot be traced back to antecedent conditions, however well these conditions may be understood (Goldstein, 2005).

However emergence is understood, it is largely impossible, due to the sheer number of interacting variables, for most of the processes involved to be tracked or observed (Goldstein, 2000). The results of the interactions also cannot be predicted, beyond certain general parameters, because it is not possible to know in advance what will interact with what, or indeed, what has interacted with what up to that point, and what has resulted from previous unknown interactions. This untrackable history of interactions (both within and beyond the system) is crucial in determining the form of future emergences, making time and history of central importance. In addition, these processes are completely decentralised, in that the order which is produced is seen to emerge solely from the multiple interactions. There is no key variable, no centrally-guiding programme or brain, and no one principle factor which makes everything happen. This does not, however, imply that anything at all can emerge, as emergence is ultimately constrained by certain features of the system itself (the idea of 'sensitive dependence on original conditions', for example, describes the effects of differing initial circumstances), and by the system's interaction with (and partial constitution by) factors and systems beyond its own boundaries (Fogel, et. al. 1997).

\section{A COMPLEXITY-BASED ONTOLOGY}

Even the simplified overview of complexity outlined above begins to gives an idea of how an ontology based on these ideas might produce some very different ways of approaching the conceptualisation and analysis of data in social research (see Byrne, 1997). Firstly, the interactions are multiple, and multiply connected, and it is the multiplicity of the interactions through time which produces effects. Causality in this situation cannot be reduced to single or limited numbers of factors, as the factors are all crucially implicated in relation to each other. Byrne has suggested that the impossibility of tracking these multiple interaction histories suggests a shift from a focus on cause to a focus on effects (Byrne, 2005b) 
Secondly, because of this connected, multi-factor causality, elements which are isolated and conceptually 'removed' from the system of connected interactions in effect cease to have meaning in terms of understanding that system (though they might have meaning in relation to other such isolated elements abstracted from other systems). The system itself has to be studied, and studied in terms of its interactions (rather than defining 'key elements' in relation to smaller units within the system and comparing these to elements from other systems). However, studying systemic interactions involves understanding that some of the interactions pertaining to the system being investigated are at the same time also interactions of other, larger/different systems which the system that is the focus of attention is embedded in and connected to.

As this kind of open system evolves through time, it is in constant 'interaction with' environmental factors, ie. factors which exist beyond its boundaries. But the language used here immediately suggests a 'thing' with a 'context'. From a dynamic systems point of view, the system, itself consisting entirely of interactions, is at any point in time partly constituted by interactions which are part of the dynamic structures of other, different systems (both larger and smaller). These other systems will have their own interaction characteristics which, in the case of larger systems, means that smaller systems within them will be sharing in the same larger system interactions. However, the interactions of larger systems are themselves constantly combining and reforming uniquely within each open system, as they combine with interactions which are particular to that system. Because all of the systems involved are conceptualised as being entirely composed of interactions, these cannot not be reduced to objects and categories, so some other way has to be found of conceptualising 'event rather than structure' (Dallmayr, 1992: 20 in Thomas, 2002: 430).

Thirdly, causality based on untrackable histories of multiple interactions means that irregularity and unpredictability have to be accepted as being a 'structural' aspect of the interactions which are to be investigated; these features cannot be 'evened out' or ignored. This unpredictability is not random 'noise' or chaos, but is part of the structuring dynamics of this type of system. The causal paths involved in multiple interactions through time may be too varied, too fast, and too simultaneous to ever be tracked or observed, but this does not mean that such causal paths are absent. It does mean, however, that researchers have to accept limits to what can be described or explained (and perhaps redirect their attention to effects, as discussed above).

Fourthly, though focussing on process, denying the existence of underpinning deep structures, and highlighting (rather than simply accommodating) unpredictability, this approach differs from post-structuralist, or post-modern critiques in offering an account of structure and also of coherence. Structure, however, here relates to processes of dynamic, de-centralised emergence, which are continually being created as a result of local interactions, and which take place in relation to constraints that exist both within and beyond the system. The idea of constraint usually implies something 'external' which operates 'on' 'something' which can be bounded, and which is at the centre of an external 'environment'. In a dynamic system, however, the constraints are as much internal to the system as they are external. Each dynamic system has a starting point in time (it emerges as a result of other sets of interactions); a specific set of 'initial conditions', and a specific and particular history of interactions through time. In this sense, every dynamic system is unique, in that even similar types of system will have emerged out of slightly different combinations of original interactions; will have 'initial conditions' that consisted of specific combinations of the many different interactions which were theoretically possible at that time and in that place; and will have specific histories which involve further particular interactions with particular combinations of factors.

Coherence, from this perspective, is not an underlying logic or set of principles which might be seen to 'underpin' different individual systems. Coherence is the existence of the system itself, and the processes which continue, through time, to maintain the system as a system. If an individual human being is thought of as a dynamic system, genetic, environmental, biological, sensory, emotional and conscious processes all continually work together to keep the person healthy, sane and socially integrated (to whatever degree...). Similarly, a social group, or an educational institution, has a coherence in the sense of a shape and an identity, although from a complexity perspective this shape and identity are in a process of continual formation, rather than resulting from essential, generative structures. 
This view of coherence indicates why it can be difficult to understand individuals when they are studied in relation to each other, or why it is difficult to apply generalised results to particular individuals. In a cross-sectional analysis, what an individual says or does at a particular point in time could be seen as an emergence, generated by an unseen history of interactions through time. As people all have different starting points, and different histories, when these emergences are compared to each other, they can appear to be messy, idiosyncratic and generally mystifying. But this is only the case when a system is observed from a vantage point which is outside of that system; if one were to try to understand the interactions, both past and present, that constitute the individual as if from within their situation, what emerges is likely to be consistent with this history of interactions (even if these cannot be tracked or measured), and with the overall impulse towards maintaining the vitality and coherence of the system.

\section{CONCEPTUALISING DIFFERENCE, SPECIFICITY AND CONTEXT}

Complexity theory challenges the nomothetic programme of universally applicable knowledge at its very heart - it asserts that knowledge must be contextual.

Byrne , 2005a

A complexity ontology provides a way of thinking about institutions, cultures, groups and individuals as systems of interactions which are, in some important ways, always unique. This uniquely presenting system of interactions, however, is partially constituted by the interactions of other, larger systems; systems of governance, for example, of culture, of language, of policy, or of funding. Other individuals, groups or institutions will be partly constituted by the same larger system interactions in the same way. Whilst the conventional approach might try to track the workings of these larger system interactions in relation to the idea of 'underpinning structures' which cut across individual examples (such as 'gender', for example), a complexity framing might suggest investigating the ways in which the aspects of these larger system interactions function within particular, smaller cases (how is this woman 'gendered' compared to this one?). The first approach privileges the similarities observable by comparison (leading to the ability to formulate a category such as 'gender' in the first place), while the complexity approach is as likely to find difference as it is to find similarity. When it does find patterns of similarity, these will relate to quite different aspects of the focus of study.

A complexity approach is helpful in relation to the problems discussed above in relation to the conceptualisation of context, and the confusion which results from the conflation of a) the context of the case with $b$ ) the contexts of the wider lives and histories of those being interviewed within the case. Thinking of people and social/institutional/cultural contexts as complex, dynamic systems allows for the separation of these two distinct types of context, even though (and perhaps crucially because) they may be embedded in each other ${ }^{5}$. If the intention is to find out something about a), the area that has been bounded as the case (ie. an Access course, a class, a school), then interviews with individuals are potentially quite limited. The researcher, attempting to 'draw together...a diverse and unrelated range of phenomena' (Hammersley \& Atkinson, 1995:211) is positioned, conceptually, 'outside' the smaller sub-units within the case (in the sense that he/she creates a category or theme which relates to narratives generated by these sub units, rather than looking at multiple elements of the case); trying to understand something that is likely to be multi-factorial in relation to a comparison of only one type of element in the dynamic system (ie. the individuals).

Gomm and Hammersley's (2001) discussion of the possible relevance of complexity theory to educational research provides an example of this kind of problem. In the study they discuss, the researchers wanted to find out why exam results were so similar over a period of time within a particular context. They point out that if the research had only interviewed examiners, producing an account of 'assessing according to the views of assessors', they could not have come to their

\footnotetext{
${ }^{5}$ For a further development of these ideas which takes account of a third type of context, that of the dynamic systems of culture and society within which the case is embedded, see Haggis, 2006
} 
final conclusion, which was that 'the selective function was smeared across the system as a whole':

...features of health visitor examining at the time, (were) to be found embedded in the selection of students, in their tuition, in the design and use of assessment instruments, in the formal talk of examination boards, in the informal talk in staff rooms, in the career trajectories of staff, and in the rich folklore there was about how to elicit - and interpret - evidence about students.

Gomm \& Hammersley, 2001:12

In order to understand how this complexity of factors worked together, over time, to produce a certain result, the researchers had to make the shift discussed above. Rather than looking from 'outside' at a collection of narratives produced by a group of separate individuals ('the view from above'), they looked as if from 'inside' what was conceptualised as a dynamically interacting system of multiple elements (the examination system within which the assessors were embedded). Although in this particular example this may seem like an obvious way of approaching the problem of understanding assessment results, it is quite a good example of the potential problem of assuming that interview narratives can 'say something' about the multiple interactions of the dynamic system within which they are embedded (although such interviews might 'say something' about how such interactions affect the individuals). This conceptualisation of the researcher looking as if from 'within' larger dynamic systems of connected factors is quite common in sociological research, but less so in many forms of small-scale educational research ${ }^{6}$.

The second problem is how to deal with b), the wider contexts (both historical and present) of the individuals who are bounded by the case. Here, the assumption is often that talking to individuals will tell the researcher something about the 'type' (in the sense of larger class) of individuals that are believed to be represented within the case. Once again, the researcher is usually conceptualised as being 'outside' the smaller sub-units within the case, and attempts to 'see' a transcendent analytical category which will hopefully indicate some kind of connection which could link to underpinning causal processes. If, however, the researcher were to shift to a position that tried to see the relevant dynamic system as if from 'inside', this would imply a need to conceptualise each person as a dynamically interacting system of multiple elements with a history through time.

As dynamic systems, each with unique starting points and histories, and yet each also partially constituted by the interactions of larger cultural and linguistic systems, individuals could, from this perspective, now be considered as systems which could justifiably be the focus of a research study. If the aim of this approach is not to identify 'deep' structural principles, and, therefore not to try to generalise in relation to such principles, then other types of connection, and of generalisation, may become possible. For example, comparisons could be made in relation to data on individuals which had be analysed longitudinally, in relation to the history and evolution of factors within that person's life, rather than at one or two points in time, in relation to factors from other people's lives.

The shift from analysing sub-units within bounded cases as if from outside such sub-units, to thinking about both the case, and its sub-components, as open systems which might be understood in relation to patterns of multiple interactions which take place within them (rather than the structures beyond them), makes it possible to examine specificity in relation to individuals from a very different ontological position. Within the dynamic system which is the case, each person might be seen as a point within a number of different interlocking dynamic systems, only one of which will be the 'case' in research terms. This 'point', however, also has its own history through time.

Thinking of individuals as dynamic systems, however, requires a reconceptualisation of 'the individual', in terms of cultural assumptions relating to ideas of an essential self or core

\footnotetext{
${ }^{6}$ Complexity and dynamic systems theories may offer a way of articulating the intentions of an ethnographic approach on the basis of a different kind of ontology.
} 
personality. The details of this are beyond the scope of this paper ${ }^{7}$. In brief, self becomes replaced by a 'sense of self', with this 'sense of self' being seen as a continually emerging property of the interactions of the system (there is no central, generative core), which functions to maintain the individual's sense of their own coherence. In the context of dynamic systems, a reconceptualised, non-essential individual begins to make space for a different understanding of both individual and context. An analysis which examines histories, traces and emergences in relation to the multiple contexts within which a 'sense of self' emerges, still, of course, employs various forms of 'reduction' and abstraction (a mistaken interpretation of complexity theory is that it somehow can 'account for' or model the totality of things [Cilliers, 1998]). This analysis reduces and abstracts, however, in relation to the history and multiplicity of each different individual, rather than by creating a transcendent category which deliberately ignores these histories and multiplicities.

\section{CONCLUSION}

This paper has attempted to use complexity thinking to draw attention to conceptual limitations of the epistemologies which underpin a large amount of qualitative research. The purpose of this is not to try to build an argument that attacks or tries to undermine these epistemologies. The discussion represents an attempt to surface some aspects of what must, inevitably, be cast into the shadows by the strong beam of established ontologies and epistemologies, and to experiment with the potential of complexity theory for suggesting different ways of thinking.

Current conceptual framings were found to problematic in relation to the conceptualisation of difference, context, processes through time, multi-factor causality, and the specificity of situations. The concept of open, dynamic systems, embedded within and partly constituting each other, whilst at the same time maintaining their own coherence, allows for different ways of thinking about context, and provides a rationale for the investigation of individuals, difference and specificity. By focusing on interactions, rather than static categories, complexity theory also makes it possible to consider different aspects of process. It does this not only in the general sense of providing a language with which to talk about dynamic interactions, but also specifically in relation to the importance of histories of interactions through time (without time, there is no emergence). In addition, complexity theory articulates a notion of causality which is multi-factorial. It is impossible to talk about isolating 'key' factors, because all of the 'factors' work together, with no one factor being more important than any other (whether or not it is helpful to even talk about 'factors' as things which 'work together' is another question). The causality implied by complexity theory is also de-centred, in the sense that there is no 'pacemaker cell' (Johnson, 2001), no gene-type entity in a dynamic system which could be said to 'cause' a particular effect, or set of effects. Causation is too multi-dimensional, too fast, and in some senses (though not others) too unpredictable to be a viable focus of attention. Complexity therefore suggests a shift from the habitual preoccupation with causes to a focus on effects (Byrne, 2005b).

The combination of multi-factor causality, occurring through time, in the absence of a central, generative force, results in the quite radical notion of emergence, and a reframing of the notion of structure. From a complexity perspective, things 'emerge' at certain points in the history of a set of multiple interactions through time, simply as a result of the interactions, rather than as the result of 'deep', generative causal structures. This is partly what makes emergence, to some extent at least, unpredictable; what emerges will depend upon what interacts, which is at least partly determined by chance encounters and changes in environments. Emergence, however, though unpredictable, is nonetheless also constrained; by features which are both internal (in terms of initial conditions and interaction histories) and external (in the sense that the system is partly made up of the interactions of larger systems, and also in the more conventional sense of physical, 'environmental' factors) to the system which is the focus of study. Though complexity theory may be seen by some as providing a way of talking about random, intuitive, even 'spiritual'

\footnotetext{
7 The idea of a dynamic and non-essential 'self' is only unusual in the context of the Western tradition (and recent 'Western' thinkers such as Derrida have, of course, worked on similar questions). Underpinned by the very different, dynamic ontology represented by the idea of 'impermanence' (which reflects many aspects of what has been discussed here in relation to complexity theory), Theravada Buddhism presents the following account of self: 'One thing disappears, conditioning the appearance of the next in a series of cause and effect... (but) there is no unchanging substance in them. There is nothing behind them that can be called a permanent self, individuality, or anything that can in reality be called 'l'. (Rahula, 1990:26)
} 
phenomena, this is not what is being argued here. The causalities involved in the interactions may be untrackable, but what emerges from them is not 'mysterious', in this sense; it is consistent with the nature and histories of the interactions involved (for discussion of a research project based on these ideas, see Haggis, 2006).

\section{REFERENCES}

Ashworth, P., \& Lucas, U. (2000). Achieving empathy and engagement: a practical approach to the design, conduct and reporting of phenomenographic research. Studies in Higher

Education, 25(3), 295-308.

Bryman, A. \& Cramer, D. (1999) Quantitative Data Analysis with SPSS Release 8 for Windows London, Routledge

Byrne, D.(2005a) Complexity, configurations and cases Theory, Culture and Society 22, 5. pp 95111

Byrne, D.(2005b) Focusing on the case in quantitative and qualitative research ESRC Research Methods Programme, Workshop 4, The Case Study January 12-13, 2005 (oral communication) Byrne, D (1998) Complexity Theory and the Social Sciences. Routledge: London Byrne, D. (1997) Complexity theory and social research Social Research Update Issue 18 pp 1-6 Cilliers, P (1998) Complexity and Postmodernism. London: Routledge

Clark, C (2005) The structure of educational research British Educational Research Journal $31,3$. pp.289-308

Flyvberg, B. (2001) Making Social Science Matter Cambridge, Cambridge University Press Furlong, J. (2004) BERA at 30. Have we come of age?, British Educational Research Journal 30, 343-358.

Fogel, A Lyra, M \& Valsiner, J (1997) Dynamics and Indeterminism in Developmental and Social Processes. New Jersey: Lawrence Erlbaum

Geertz, C. (1973) On thick description in C. Geertz, The interpretation of culture New York, Basic Books

Giere, R. (2000) Theories in A companion to the philosophy of science Oxford, Blackwell Goldstein, J. (2005) Impetus without teleology: The self-transcending construction of emergence Paper given at the Complexity, Science and Society Conference, Liverpool, September 2005

Goldstein, J (2000) Emergence: A Construct Amid a Thicket of Conceptual Snares, in: Emergence 2 (1): 5-22

Gomm, R. \& Hammersley, M. (2001) Thick ethnographic description and thin models of complexity Paper presented at British Educational Research Association Annual Conference, Leeds University, 13-15 September

Goodwin, L. (2002) Resilient Spirits London, Routledge Falmer

Guba, E \& Lincoln, Y (1998) Competing Paradigms in Qualitative Research, in: N. Denzin \& Y. Lincoln, The Landscape of Qualitative Research. London: Sage

Haggis, T. (2006) Context, agency and time: looking at learning from the perspective of complexity and dynamic systems theory. Paper presented at ESRC Teaching and Learning Research Programme Thematic Seminar Series: Contexts, communities, networks; Mobilising learner's resources and relationships in different domains, University of Stirling, June 2006 Hammersley, M. (1989) The Dilemma of Qualitative Method London, Routledge Hammersley, M. (2005) Review of Key Themes in Qualitative Research: Continuities and Change British Educational Research Journal 31, 3. pp405-408

Hargreaves, D. (1996) Teaching as a research-based profession London, Teacher Training Agency

Hollis, M. (2003) Explaining and understanding in The Blackwell Companion to Philosophy

Oxford, Blackwell

Hammersley, M. \& Atkinson, P. (1995) Ethnography $2^{\text {nd }}$ Edition (London, Routledge).

Johnson, S (2001) Emergence. London: Penguin

Law, J. \& Urry, J, (2003) 'Enacting the Social', published by the Department of Sociology and the Centre for Science Studies, Lancaster University, Lancaster LA1 4YN, UK, at http://www.lancs.ac.uk/fss/sociology/research/resalph.htm\#lr

Llewelyn, S. (2003) What counts as 'theory' in qualitative management and accounting research? Accounting, Auditing and Accountability Journal 16, p 662-708 
Marton, F. Hounsell, D. Entwistle, N. (eds) (1997) The experience of learning Edinburgh, Scottish academic press

Moore, L., Graham, A. \& Diamond, I. (2003) On the feasibility of conducting randomised trials in education: case study of a sex education intervention British Educational Research Journal 29, 5. pp. 673-689

Nespor, J. (1994) Knowledge in Motion: Space, time and curriculum in undergraduate physics and management London, Falmer

Newton, J. Implementing a teaching and learning strategy Studies in Higher Education (2003) 28.4

Osberg, D. (2002) Complexity, science and education: some insights and possibilities Paper presented at the $32^{\text {nd }}$ SCUTREA Annual Conference, University of Stirling, 2-4 $4^{\text {th }}$ July

Osberg, D. (2005) Curriculum, Complexity and Representation Unpublished PhD Thesis, The Open University, Milton Keynes

Rahula, W. (1990) What the Buddha Taught London, The Gordon Fraser Gallery

Roberts, B. (2002) Biographical Research Buckingham, Open University Press

Rock, P. (1973) Phenomenalism and essentialism in the sociology of deviance Sociology 7,1 pp.17-29

Richardson, K. \& Cilliers, P. (2001) What is complexity science? A view from different directions

Emergence 3, 1. pp 5-22

Seibt, J. (2003) (ed) Process Theories London, Kluwer

Silverman, D. (2001) Interpreting Qualitative Data London, Sage ( $2^{\text {nd }}$ edition)

Smelser, N.J. \& Bates, P.B., eds. (2001) International Encyclopedia of the Social \& Behavioral Sciences. Oxford, UK: Pergamon

Stein, S. Isaacs, G. \& Andrews. T. (2004) Incorporating authentic learning experiences within a university course Studies in Higher Education 29, 2. pp237-258

Stehr, N. \& Grundmann, R. (2001) The authority of complexity British Journal of Sociology 52, 2 pp. 313-329

Strauss, A. \& Corbin, J. (1998) Basics of Qualitative Research Thousand Oaks, Sage

Strathern, M. (1992) Partial Connections, Rowman \& Littlefield, London

Thomas, G. (2002) Theory's spell - on qualitative inquiry and educational research British Educational Research Journal 28, 3. pp. $419-435$

Tooley, J. \& Darby, D. (1998) Educational Research: a critique London, Office for Standards in Education.

Unwin, L., et al (2005) Worlds within worlds: the relationship between context and pedagogy in the workplace Paper presented at ESRC Teaching and Learning Research Programme Thematic Seminar Series: Contexts, communities, networks: mobilizing learner's resources and relationships in different domains

Valsiner, J (1998) The Guided Mind. Massachusetts: Harvard University Press

Yanow, D. (2003) Interpretive empirical political science: what makes this not a subfield of qualitative methods Qualitative Methods Section (APSA) Newsletter $2^{\text {nd }}$ Issue 
